\title{
Motivation and Local Image Entropy Based Measures in Evolutionary Mobile Robot Navigation
}

\author{
Tomás Arredondo and Wolfgang Freund \\ Universidad Técnica Federico Santa María \\ Chile
}

\section{Introduction}

Robotic navigation in unknown and unstructured environments is a complex and difficult task fraught with potential problems and peril to the navigator. Extensive research has been made and is ongoing into this problem with the aid of various robotic architectures, sensors and processors. Behavior based robotics is an approach that in general does not promote the use of complex world models or symbolic knowledge. This design philosophy promotes the idea that robots should be low cost, built incrementally, capable of withstanding sensor and other noise, and without complex computers and communication systems. Behavior based learning systems typically include reinforcement learning, neural networks, genetic algorithms, fuzzy systems, case and memory based learning (1). These biologically based mechanisms are capable of novel complex behaviors which avoid local minima and have the ability to extrapolate from training information.

One area of research in behavior based robotics has focused on providing more natural and intuitive interfaces between robots and people $(2 ; 3)$. One recent investigation (4), decouples specific robot behavior using an intuitive interface based on biological motivations (e.g. curiosity, hunger, etc) (5). Training the robot to behave according to said motivations requires optimization of the robot inference system (e.g. neural network) which in our approach is implemented using a genetic algorithm (GA).

It is a well known fact in machine learning (6), that having diversity during training can provide for the emergence of more robust systems which are capable of coping with a variety of environmental challenges $(7 ; 8)$. Early studies have shown that information theory can be used as an aid in analyzing robotic learning performance in terms of the diversity of information received during training $(4 ; 9)$. Performing a more extensive analysis of environment training diversity using such information theoretic based measures was something of interest to us. Toward this goal we investigate the capability of the entropy based environmental and motivation measures toward analyzing the outcome of several robotic navigation experiments.

In our work, robot navigation is performed in a simulator (10) by providing sensor values directly into a neural network inference engine that drives left and right motors. The robot uses infrared sensors which give limited information about the surroundings in which the robot is located. In order to reduce the complexity of the action space, action-based environmental modeling (AEM) (11) is implemented with a small action set of four basic actions (e.g. go straight, turn left, turn right, turn around) in order to encode a sequence of actions based 
on sensor values. The search space of behaviors is huge and designing suitable behaviors by hand is very difficult (11) therefore we use a genetic algorithm (GA) within the simulator in order to find appropriate behaviors. The GA selects from a population of robots (neural networks) using a fuzzy fitness function that considers various robotic motivations such as: the need for exploration (curiosity), the need to conserve its battery (energy), the desire to determine its location (orientation), and the capacity to return to its initial position (homing). This paper is organized as follows. Section 2 gives a description of the robotic system. Section 3 describes the entropy measures used for diversity evaluation. Section 4 introduces the experiments performed. In section 5 we describe and summarize our results. Finally, in section 6 some conclusions are drawn.

\section{Robotics System Description}

This section presents the robotic system used for these studies. The system has several different elements including: the robot simulator, action based environmental modeling, neural networks, GA, and fuzzy logic based fitness (Fig.1).

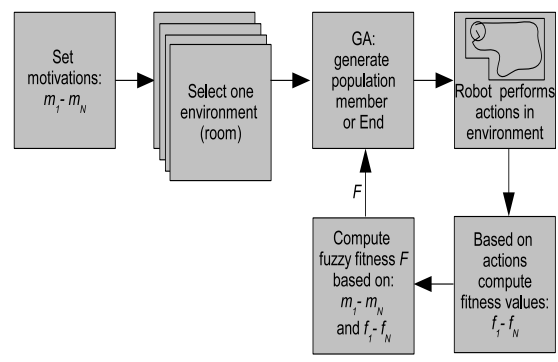

Fig. 1. System Overview

\subsection{Robot Configuration}

Our project uses a small mobile robot which has two DC motors and eight (six front and two back) infrared proximity sensors used to detect nearby obstacles. These sensors provide 10 bit output values (with $6 \%$ random noise). These readings allow the robot to know in approximate form the distance to local obstacles. The simulator (10) provides the readings for the robot sensors according to the robot position and the map (room) it is in. The simulator also has information for the different areas that the robot visits and the various obstacles detected in the room.

The robot internally generates a zone map in which the zones are marked with various values: obstacles are indicated with a value of -1 , those not visited by the robot are marked with a 0 and the visited ones with a 1. During navigation, the robot executes 500 steps in each experiment, but not every step produces forward motion as some only rotate the robot. The robot is not constrained by its battery given that a 100\% charge level allows more than 1000 steps.

\subsection{Artificial Neural Network}

As seen in (Fig.2), the robot uses a feed-forward neural network with eight input neurons (one per sensor), five neurons in the hidden layer and two output neurons directly connected to the 
motors that produce the robot movement. The transfer function used by the neurons is of the sigmoid type.

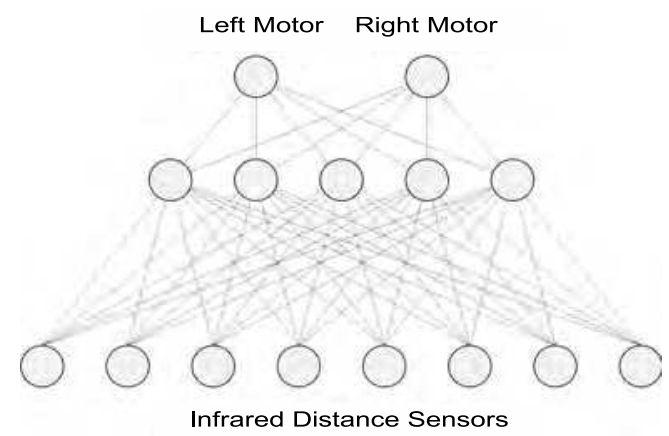

Fig. 2. Neural Network

\subsection{Action-based Environmental Modeling}

In order to reduce the vast behavior search space, we use AEM (11) based encoding to select the basic actions used by the robot to perform navigation:

- A1: Go $55 \mathrm{~mm}$ straight on.

- A2: Turn $30^{\circ}$ left.

- A3: Turn $30^{\circ}$ right.

- A4: Turn $180^{\circ}$ right.

AEM uses a SOM (12) network so that the robot may determine the room it is navigating in (localization). Robot navigation produces actions which are saved as action sequences. These action sequences are converted using chain coding into an environment vector. These vectors are fed into the SOM network for unsupervised learning. After training the SOM network associates a vector with one of its output nodes ( $r$-nodes)(11). We used inputs of 1000 steps for all rooms used in training, these were alternatingly presented to the SOM network for 10000 iterations, the network had a linear output layer of $128 r$-nodes indicating the maximum possible number of rooms that could be identified.

\subsection{Genetic Algorithm}

A GA is used to find an optimal configuration of weights for the neural network. Each neural network is encoded as an individual in the GA by a concatenation of all the weights in the neural network. The GA evolves the neural network with the passing of different generations using the fuzzy fitness measure described below (Fig.3). The GA uses the following parameters:

- Population size: 200.

- Crossover operator: Random crossover.

- Selection method: Elite strategy selection.

- Mutation rate $P_{m u t}: 1 \%$.

- Generations: 90. 


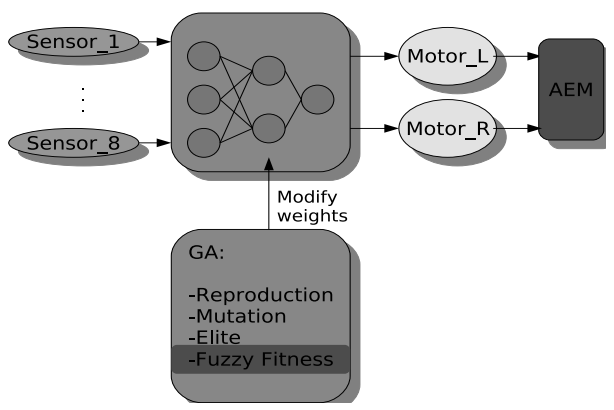

Fig. 3. GA Overview

\subsection{Fuzzy Fitness Calculation}

Fuzzy logic (Fig.4) is used toward implementing a motivation based interface and towards determining the fitness of robotic behavior. The motivation set $(M)$ considered includes: homing $\left(m_{1}\right)$, curiosity $\left(m_{2}\right)$, energy $\left(m_{3}\right)$, and orientation $\left(m_{4}\right)$. These motivations are used as input settings (between 0 and 1 ) prior to running each experiment (4).

The set of fitness criteria and the fuzzy variables that correspond to them are: proper action termination and escape from original neighborhood area $\left(f_{1}\right)$, amount of area explored $\left(f_{2}\right)$, percent of battery usage $\left(f_{3}\right)$ and environment recognition $\left(f_{4}\right)$. The values for these criteria are normalized (range from 0 to 1 ) and are calculated after the robot completes each run:

- $f_{1}$ : a normalized final distance to home

- $f_{2}$ : percentage area explored relative to the optimum

- $f_{3}$ : estimated percent total energy consumption considering all steps taken

- $f_{4}$ : determined by having the robot establish which room he is in ( $r$-node versus the correct one), using the previously trained SOM network.

Four fuzzy variables with five triangular membership functions each are used $\left(5^{4}=625\right.$ different fuzzy rules) toward calculating robotic fitness. The membership functions used are given in Fig. 4. Sample fuzzy rules (numbers 9 and 10) are given as follows (here the K array is a simple increasing linear function):

if $\left(f_{1}==\mathrm{H}\right)$ and $\left(f_{2}==\mathrm{L}\right)$ and $\left(f_{3}==\right.$ V.L. $)$ and $\left(f_{4}==\right.$ V.L. $)$ then

$\mathrm{f}[9]=m_{1} f_{1} K[4]+m_{2} f_{2} K[2]+m_{3} f_{3} K[1]+m_{4} f_{4} K[1]$

if $\left(f_{1}==\right.$ V.H. $)$ and $\left(f_{2}==\mathrm{L}\right)$ and $\left(f_{3}==\right.$ V.L. $)$ and $\left(f_{4}==\right.$ V.L. $)$ then

$\mathrm{f}[10]=m_{1} f_{1} K[5]+m_{2} f_{2} K[2]+m_{3} f_{3} K[1]+m_{4} f_{4} K[1]$

During training, a run environment (room) is selected and the GA initial robot population is randomly initialized. After this, each robot in the population performs its task (navigation and optionally environment recognition) and a set of fitness values corresponding to the performed task are obtained. Finally, robotic fitness is calculated using the fitness criteria information provided by the simulator and the different motivations at the time of exploration. 


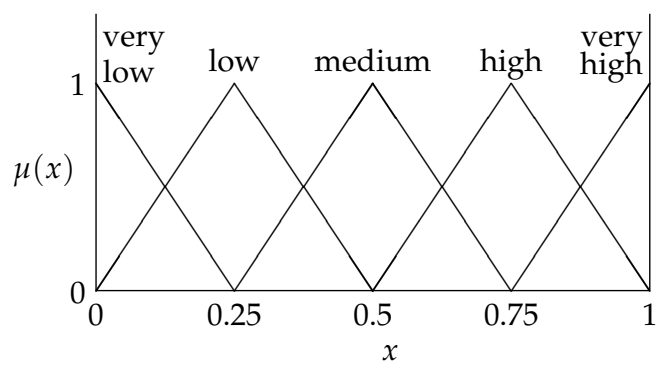

Fig. 4. Fuzzy membership functions

\section{Entropy Measures}

Two entropy based measures are used in this study to measure environmental and motivation diversity (4). These measures of diversity come from the well established definition of entropy as a measure of the uncertainty (which generates a diversity of outcomes) in a system $(13 ; 14)$.

\subsection{Environmental Entropy}

Average local image entropy is used as a measure of environmental uncertainty (i.e. uncertainty being a measure of an environmental diversity) given the shape and distribution of obstacles in a room by using an extension of the local image entropy method (4). In the local image entropy method an estimation of the information content of a pixel $(x, y)$ based on a histogram of its neighborhood $(w, h)$ pixel values is calculated as:

$$
E_{w, h}(x, y)=-\sum_{k} p_{w, h}(k) \log p_{w, h}(k)
$$

A region of an image (e.g. neighborhood) is interpreted as a signal of $k$ different states with the local entropy $E_{w, h}(x, y)$ determining the observer's uncertainty about the signal (15).

To obtain a measure of an environment's diversity (using a neighborhood window as given by $w$ and $h$ ), we compute the average local image entropy for the room. Here the size of $w$ and $h$ are set using an uncertainty criteria of interest given the scale of the robot and obstacles in its path (e.g. a robots uncertainty of crossing a zone). In our experiments we study this diversity measure to find neighborhood width and height settings which are successful in helping us predict better training environment sets.

Clearly if a neighborhood is empty or full the robot will have either no difficulty or no chance of traversing the terrain and hence the uncertainty for that neighborhood will be zero but if the neighborhood has some obstacles then its maximum uncertainty should be when $E_{w, h}(x, y)=$ 1.0 .

Obstacles were chosen at least as large as the robot size so as to preclude small checkered patterns which could also result in a local entropy value near 1.0 but would not make any sense in terms of our definition of traversing uncertainty (the robot clearly could not cross such a neighborhood).

\subsection{Motivation Entropy}

In order to calculate motivation entropy we first define a motivation set $M$ as $\left\{m_{1}, m_{2}, \ldots, m_{n}\right\}$. Toward the calculation of motivation diversity $H(M)$, we consider the cor- 


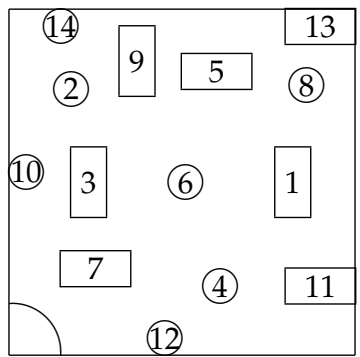

Fig. 5. Experiment 1 room layout $\left(r_{1}-r_{15}\right)$.

responding probabilities for $\left\{m_{1}, m_{2}, \ldots, m_{n}\right\}$ as $\left\{p_{1}, p_{2}, \ldots, p_{n}\right\}$. We compute the entropy of the random variable $M$ using:

$$
H(M)=-\sum_{i=1}^{n} p_{i} \log \left(p_{i}\right), \text { where } \sum_{i=1}^{n} p_{i}=1 .
$$

Note that when $H(M)=0$ the motivations are considered to have no diversity and for $n=4$ the maximum diversity is when $M=\{0.25,0.25,0.25,0.25\}$ and $H(M)=2$.

\section{Experimental Evaluation}

Of interest to us was to study the impact of the entropy based diversity measures and their capability toward predicting a robot's ability to learn behaviors based on environmental and motivation diversity. Our results were verified based on an empirical model using multiple complete test runs given random motor and sensor noise.

All experiments had a training phase, in order to obtain the best weights for the NN, followed by a testing phase. The GA settings used in the training phase are given in section 2.4. The number of steps in each iteration was set to 1000 for the training phase, and 500 for the testing phase. The robot radius was $68.75 \mathrm{~mm}$ with a forward step size of $55 \mathrm{~mm}$. The rooms are square (sides of $2750 \mathrm{~mm}$ ) with various internal configurations of walls and obstacles. For mapping purposes rooms are divided into 2500 zones (each $55 \mathrm{~mm}$ by $55 \mathrm{~mm}$ ).

Toward studying the diversity measures, we tested training in 15 different square rooms: $r_{1}$ $r_{15}$. These rooms have an increasing number of obstacles in a fairly random distribution with the condition that any one obstacle should not preclude the robot from reaching any area of the room. Room $r_{1}$ has no obstacles, $r_{2}$ has only obstacle $1, r_{3}$ has obstacle 1 (in the same place as in $r_{2}$ ) plus obstacle 2 , and so on until $r_{15}$ which contains obstacles $1-14$. A room layout with the position of the obstacles (identified by its number) is shown in Fig. 5.

\subsection{First experiment: environmental diversity measure}

In order to analyze the sensitivity of the environmental diversity metric we first computed the average local image entropy of each training room given different square window sizes (given as $w$ or $h$ ). These values are seen in Fig. 6 which shows the average local entropy of each room given a range of window sizes from 0 to 1000.

To evaluate the impact of environmental training diversity over the robots navigation behavior, we trained the robot in each room for 90 generations. We set the fuzzy motivations 


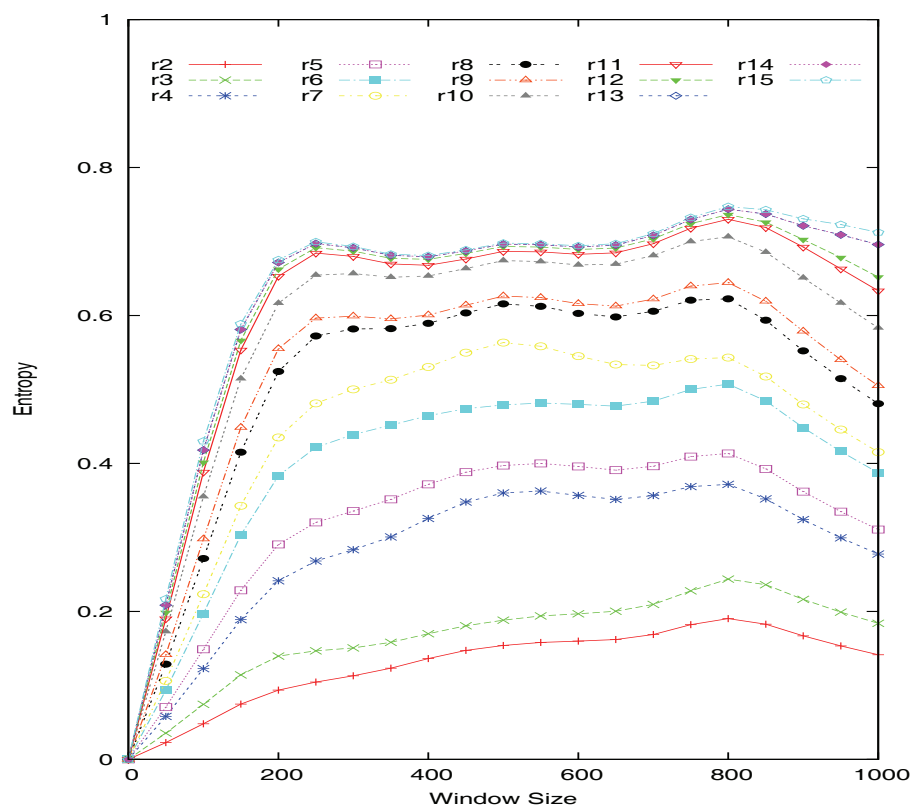

Fig. 6. Average local entropy for all rooms.

$\left(m_{1}, m_{2}, m_{3}, m_{4}\right)$ as $(0,1,0,0)$. After the training phase, the best individual from the GA population was selected to run its respective testing phase in rooms $r_{1}-r_{10}$. During testing, rooms $r_{11}-r_{15}$ produced low fitness in all experiments, because of this small contribution we discarded these results from our analysis. A window size of 100 was used given that it is the minimum window size which can still discern changes in the environment and provides the best small region granularity (after analyzing window size results in Fig.6). Even so other possible window sizes could be used for example a window size of 200 would give greater separation between rooms but lower granularity.

\subsection{Second experiment: motivation diversity measure}

For this experiment, in order to see the effect of motivation diversity, we used 66 sets of fuzzy motivation criteria. Motivations $(M)$ ranged from highly focused to more diverse. Average fitness values are given for motivations $\left(m_{1}, m_{2}, m_{3}\right)$ using all combinations ranging from $(0,0,1)$ to $(0.4,0.3,0.3)$ with values changing in increments of 0.1 . Minimum entropy for $M$ is 0 and maximum entropy is 1.571

The population was trained for 90 generations in each of the rooms. During testing the best individual of the 90 generations was tested in all rooms and average fuzzy fitness values were calculated using the various fitness values $f_{1}-f_{3}$. 


\section{Experimental Results}

Ten complete runs were performed of each experiment (each run consisting of one training and 10 test executions) and only average values are reported in our results. Only ten runs are used given that obtained tests results were similar between runs.

\subsection{First experiment: environmental diversity}

In Fig.7 we show the results of the testing phase after applying the respective training method specified for the environmental diversity experiment.

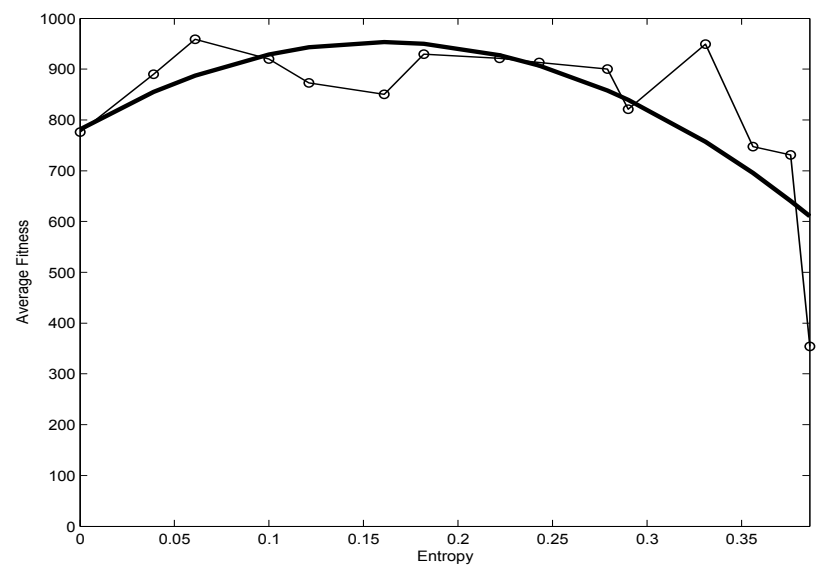

Fig. 7. Learning behavior given training rooms entropy.

\subsection{Second experiment: motivation diversity}

In Fig. 8 we show the results of the testing phase after applying the respective training method specified for the motivation diversity experiment.

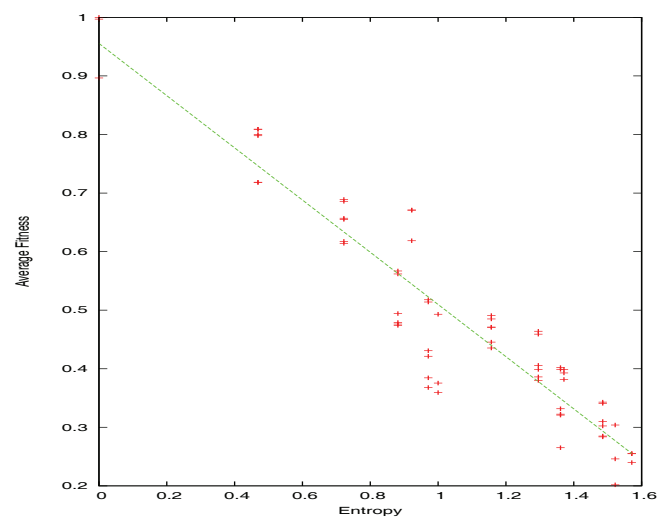

Fig. 8. Learning behavior given motivation diversity. 


\section{Discussion}

Average local image entropy can be seen as an effective measure of a training environments potential toward producing highly fit robots. As seen in Fig.6 the measure is sensitive to different window values but in our application a wide range of values could have been used. Our selection of neighborhood size was a reasonable as only very low values (around 10) show poor discriminancy.

Fig. 7 shows how the average fitness obtained during testing is clearly dependent on the diversity of the training room used. A training environment with too much environmental diversity is as unsuitable as one with not enough diversity.

Higher motivation diversity $(H(M))$ caused lower average score values. This is possibly due to the different and conflicting (e.g. orthogonal) requirements of the different motivations upon the robotic neural brain. These scores are also negatively impacted by the fitness function used (4) given that it includes a motivation satisfaction product $\left(m_{n} \times f_{n}\right)$ in each rule that further penalizes lower behavior fitnesses $\left(f_{n}\right)$. Even though obtained fitness was generally lower with more diverse motivations, the obtained behaviors demonstrated very good capability (e.g. room exploration, battery usage, etc) and were in agreement with the specified motivations. Because of this, motivation diversity results are somewhat counter intuitive in that by diversifying motivation values one could naively expect higher overall fitness but this is clearly not the case due to system constraints.

\section{Conclusions}

Our entropy based measures are useful toward providing some understanding of complex systems such as robotic training environments. As seen by our test results, the average local image entropy measure is not very sensitive to differences in window size as long as they are above a minimum value. In general the window size used should be the minimum value possible that can still differentiate between environments. The average local entropy measure also shows promise as an image diversity metric with potential usage in signal processing and pattern recognition. Fuzzy motivations are confirmed as an intuitive and user friendly mechanism for obtaining a wide range of different robotic behaviors. Using these entropy based measures it was possible to evaluate the effect of diversity on robotic fitness.

Future work includes hardware implementation and testing of these methods, testing other fuzzy fitness functions, utilizing fuzzy motivations within hybrid architectures, including environment recognition in motivation studies, parametric studies (e.g. linkages between motivations, testing different groups of GA parameters), adding different actions to the robot and potential application in other areas of research.

\section{Acknowledgements}

This research was partially funded by the DGIP of UTFSM (230808).

\section{References}

[1] Arkin, R.: Behavior-Based Robotics, MIT Press, Cambridge, (1998).

[2] Park, H., Kim, E., Kim, H.: Robot Competition Using Gesture Based Interface. LNAI,Vol. 3353. Springer-Verlag, Berlin (2005) 131-133 
[3] Jensen, B., Tomatis, N., Mayor, L., Drygajlo, A., Siegwart, R.: Robots Meet Humans Interacion in Public Spaces. IEEE Transactions on Industrial Electronics, Vol. 52, No. 6 (2006) 1530-1546

[4] Arredondo, T., Freund,W., Muñoz, C., Navarro, N., and Quirós, F.: Learning Performance in Evolutionary Behavior Based Mobile Robot Navigation. LNAI, Vol. 4872. SpringerVerlag, Berlin (2007) p. 811-820

[5] Huitt, W.: Motivation to learn: An overview. Educational Psychology Interactive. http:/ /chiron.valdosta.edu/whuitt/col/motivation/motivate.html, (2001).

[6] Mitchell, T.: Machine Learning, McGraw Hill, Boston, (1997)

[7] Tan, K.C., Goh, C.K, Yang, Y.J., Lee, T.H.: Evolving better population distribution and exploration in evolutionary multi-objective optimization, European Journal of Operations Research 171 (2006) 463-495

[8] Chalmers, D.J.: The evolution of learning: An experiment in genetic connectionism. Proceedings of the 1990 Connectionist Models Summer School, p. 81-90. San Mateo, CA.: M. Kaufmann, 1990

[9] Arredondo, T., Freund,W., Muñoz, C.: Entropy Based Diversity Measures in Evolutionary Mobile Robot Navigation. LNCS, Vol. 5027. Springer-Verlag, Berlin (2008) p. 129-138

[10] YAKS simulator website: http://www.his.se/iki/yaks

[11] Yamada,S.: Recognizing environments from action sequences using self-organizing maps, Applied Soft Computing, 4 (2004) 35-47

[12] Teuvo, K.: The self-organizing map. Proceedings of the IEEE, 79(9), (1990) 1464-1480

[13] Cover, T., Thomas, J.: Elements of Information Theory, Wiley, New York, (1991)

[14] Neelakanta, P. S.: Information-Theoretic Aspects of Neural Networks, CRC Press, Boca Raton, (1999)

[15] Handmann, U., Kalinke, T., Tzomakas, C., Werner, M., Weelen, W.v.,: An image processing system for driver assistance. Image and Vision Computing, 18, (2000) 367-376 


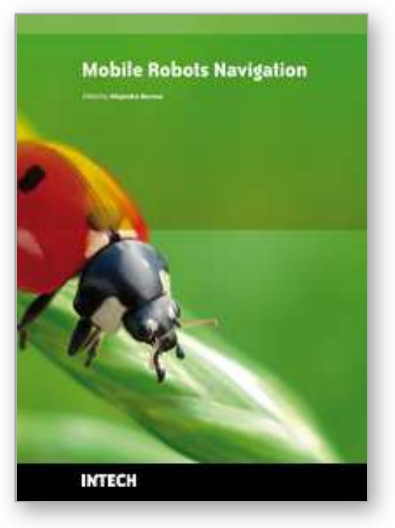

\author{
Mobile Robots Navigation \\ Edited by Alejandra Barrera
}

ISBN 978-953-307-076-6

Hard cover, 666 pages

Publisher InTech

Published online 01, March, 2010

Published in print edition March, 2010

Mobile robots navigation includes different interrelated activities: (i) perception, as obtaining and interpreting sensory information; (ii) exploration, as the strategy that guides the robot to select the next direction to go; (iii) mapping, involving the construction of a spatial representation by using the sensory information perceived; (iv) localization, as the strategy to estimate the robot position within the spatial map; (v) path planning, as the strategy to find a path towards a goal location being optimal or not; and (vi) path execution, where motor actions are determined and adapted to environmental changes. The book addresses those activities by integrating results from the research work of several authors all over the world. Research cases are documented in 32 chapters organized within 7 categories next described.

\title{
How to reference
}

In order to correctly reference this scholarly work, feel free to copy and paste the following:

Tomas Arredondo and Wolfgang Freund (2010). Motivation and Local Image Entropy Based Measures in Evolutionary Mobile Robot Navigation, Mobile Robots Navigation, Alejandra Barrera (Ed.), ISBN: 978-953-307076-6, InTech, Available from: http://www.intechopen.com/books/mobile-robots-navigation/motivation-andlocal-image-entropy-based-measures-in-evolutionary-mobile-robot-navigation

\section{INTECH}

open science | open minds

\section{InTech Europe}

University Campus STeP Ri

Slavka Krautzeka 83/A

51000 Rijeka, Croatia

Phone: +385 (51) 770447

Fax: +385 (51) 686166

www.intechopen.com

\section{InTech China}

Unit 405, Office Block, Hotel Equatorial Shanghai

No.65, Yan An Road (West), Shanghai, 200040, China

中国上海市延安西路65号上海国际贵都大饭店办公楼 405 单元

Phone: +86-21-62489820

Fax: +86-21-62489821 
(C) 2010 The Author(s). Licensee IntechOpen. This chapter is distributed under the terms of the Creative Commons Attribution-NonCommercialShareAlike-3.0 License, which permits use, distribution and reproduction for non-commercial purposes, provided the original is properly cited and derivative works building on this content are distributed under the same license. 